\title{
Research Note \\ Mass accretion rate of the galactic black hole A0620-00 in its quiescent state
}

\author{
S. Pal ${ }^{1}$ and S. K. Chakrabarti ${ }^{1,2}$ \\ ${ }^{1}$ Centre for Space Physics, Chalantika 43, Garia Station Rd., Kolkata, 700084, India \\ 2 S. N. Bose National Centre for Basic Sciences, Salt Lake, Kolkata, 700098, India \\ e-mail: chakraba@bose.res.in
}

Received 24 October 2003 / Accepted 14 March 2004

\begin{abstract}
Our recent radio observation using the Giant Meter Radio Telescope (GMRT) of the galactic black hole transient A0620-00 at 1.280 GHz revealed a micro-flare of a few milli-Jansky. Assuming an equipartition of magnetic energy and the gravitational potential energy of accreting matter, it is possible to estimate the upper limit of the accretion rate. Assuming a black hole mass of $10 M_{\odot}$ residing at the center, we find this to be at most $\dot{M}=(8.5 \pm 1.4) \times 10^{-11} M_{\odot} \mathrm{yr}^{-1}$. This is consistent with earlier estimates of accretion rates based on optical and X-ray observations.
\end{abstract}

Key words. black hole physics - accretion, accretion disks - magnetic fields - radio continuum: general techniques: photometric - methods: observational

\section{Introduction}

The galactic black hole transient A0620-00 is not particularly well known for its activity in radio wavelengths. It was last reported to have radio outbursts in 1975 at 962 and $151 \mathrm{MHz}$ (Davis et al. 1975; Owen et al. 1976). A few years after this observation, Duldig et al. (1979) reported a low level activity at $2 \mathrm{~cm}(14.7 \mathrm{GHz})$. More recent re-analysis of the 1975 data revealed that it underwent multiple jet ejection events (Kuulkers et al. 1999). There are no other reports of radio observations of this object.

A0620-00 is in a binary system and its mass is estimated to be around $10 M_{\odot}$ (Gelino et al. 2001). It was discovered in 1975 through the Ariel V sky survey (Elvis et al. 1975). This object is located at a distance of $1.05 \mathrm{kpc}$ (Shahbaz et al. 1994). The outbursts and quiescence are thought to be due to some form of thermal-viscous-instability in the accretion disk. In the quiescent state, the accretion rate becomes very low (e.g., Lasota 2001). Assuming there is a Keplerian disk, from optical and X-ray observations the accretion rate was estimated to vary from a few times the Eddington rate in outbursts to less than $10^{-11} M_{\odot} \mathrm{yr}^{-1}$ in quiescence (de Kool 1988; McClintock \& Remillard 1986). Assuming a low-efficiency flow model, McClintock \& Remillard (2000) obtained the accretion rate to be $\sim 10^{-10} M_{\odot} \mathrm{yr}^{-1}$ using X-ray observations. A0620-00 has

Send offprint requests to: S. Pal, e-mail: space_phys@vsnl.com been in a quiescent state for quite some time. Our understanding of the accretion processes at low accretion rates suggests that the magnetic field may be entangled with hot ions at virial temperatures and could be sheared and amplified to the local equipartition value (Rees 1984). If so, dissipation of this field, albeit small, should produce micro-flares from time to time, and they could be detectable especially if the object is located nearby.

In the present Paper, we report the observation of a microflare in radio wavelength (frequency $1.28 \mathrm{MHz}$ ) coming from this object. In the next section, we present the details of the observations and the results. In Sect. 3, we analyze our observation and compute the accretion rate in the quiescent state.

\section{Observations and results}

On Sep. 29th, 2002, from UT 00:45-02:03 we observed A0620-00 with the Giant Meter Radio Telescope (GMRT) located in Pune, India. GMRT has 30 parabolic reflector antennae placed on altazimuth mounts each of which is of $45 \mathrm{~m}$ diameter. During our observation, 28 of 30 antennae were working and the observational conditions were stable. It has a tracking and pointing accuracy of $1^{\prime}$ for wind speeds less than $20 \mathrm{~km} \mathrm{~s}^{-1}$. GMRT is capable of observing at six frequencies from $151 \mathrm{MHz}$ to $1420 \mathrm{MHz}$. On the higher side, 608-614 MHz and 1400-1420 MHz are protected frequency bands by the International Telecommunication Union (ITU). 


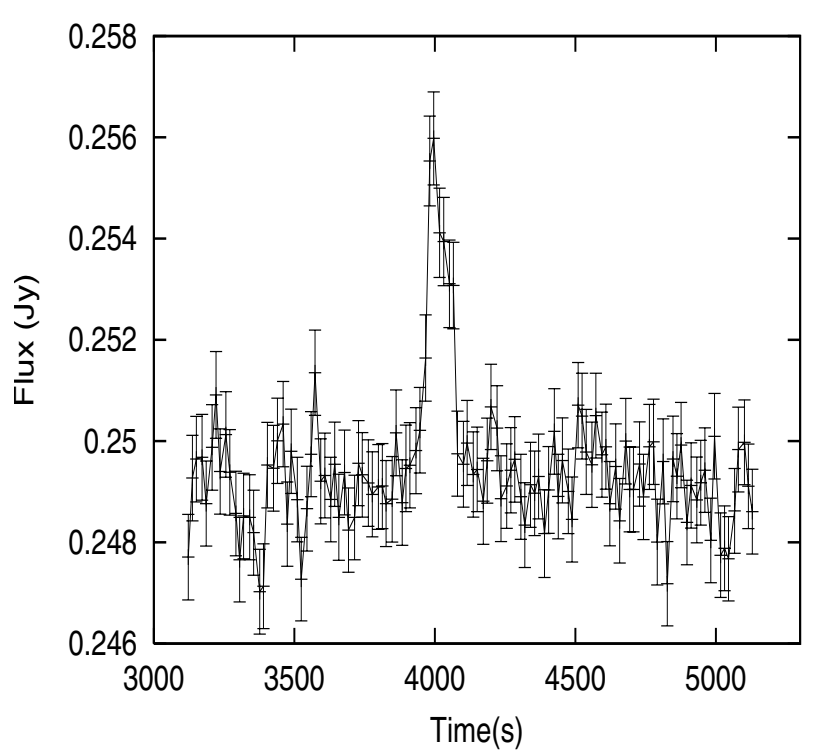

Fig. 1. Lightcurve of A0620-00 without background subtraction on Sep. 29th, 2002 as obtained by GMRT radio observation at $1.28 \mathrm{GHz}$. Subtracting the background reveals a micro-flare of mean flux 3.84 mJy of duration $192 \pm 32 \mathrm{~s}$.

The observed frequency is $v_{\text {obs }} \sim 1280 \mathrm{MHz}$ far away from the ITU bands. The band width is $16 \mathrm{MHz}$. There were 128 channels with a channel separation of $125 \mathrm{kHz}$. The light-curve without the background subtraction is shown in Fig. 1. The data is saved every $16 \mathrm{~s}$. The background is due to two side lobes and is found to be constant in time. The UV coverage was very good and the background was found to be constant within the field of view with rms noise $8.6 \times 10^{-4} \mathrm{Jy}$ as tested by the task IMAGR in AIPS. The UVRANGE task required no constraint. The background subtraction reveals that a microflare of average flux density of $F_{v}=3.84 \mathrm{mJy}$ occurred and it lasted for about $t_{\mu f}=192 \pm 32 \mathrm{~s}$. We used 3C 147 as the flux calibrator and $0521+166$ as the phase calibrator. No other source was found within the field of view. The primary beam width is 0.5 degree and the synthesized beam width is 3 arcsec. The confirmation of this micro-flare is shown by the fact that each of the antennae independently recorded this event and the synthesized image of the rest of the field of view showed no significant signal.

\section{Discussion}

Close to a black hole, fast variabilities in time scales of the order of the light crossing time $t_{1}=r_{\mathrm{g}} / c \sim 0.1 \frac{M}{10 M_{\odot}} \mathrm{ms}$, $r_{\mathrm{g}}=2 G M / c$ is the Schwarzschild radius, is possible. Shot noise in this time scale is observed during X-ray observations. The duration $t_{\mu \mathrm{f}}$ of the micro-flare that we observe is much larger $\left(t_{\mu \mathrm{f}} \gg t_{1}\right)$, and hence we rule out the possibility that it is a shot noise type event.
Assuming that the flare is due to magnetic dissipation, with an energy density of $B^{2} / 8 \pi$, the expression for the total energy release (fluence) is:

$E_{\mathrm{mag}}=\frac{B^{2}}{8 \pi} V=4 \pi D^{2} v_{\mathrm{obs}} F_{v} t_{\mu \mathrm{f}}$,

where $V \sim r_{\mathrm{g}}^{3}$ is the lower limit of the volume in the accretion flow that released the energy, $D$ is the distance of the source from us, $v_{\mathrm{obs}}$ is the frequency at which the observation is made and $F_{v}$ is the specific intensity of radiation. Here, $B$ is the average magnetic field in the inflow where the flare forms. Rewriting Eq. (1) using the equipartition law,

$$
\frac{B^{2}}{8 \pi} \sim \frac{G M \rho}{r}=\frac{G M \dot{M}}{4 \pi v r^{3}}
$$

where $\rho$ is the density of the flow in the accretion flow, $\dot{M}$ is the accretion rate and $v$ is the velocity of inflow. Since there is no signature of a Keplerian disk in the quiescent state, one may assume the inflow to be generally like a Bondi flow (Chakrabarti 1990), especially close to the black hole. Estimations of McClintock \& Remillard (2000) were carried out with a low-efficiency radial flow model. Thus we use the definition of the accretion rate to be $\dot{M}=4 \pi \rho r^{2} v$. More specifically, we assume the free-fall velocity, $v \sim(2 G M / r)^{1 / 2}$. Introduction of pressure and rotation effects do not change the result since the gas is tenuous, and since the Keplerian flow is absent, the angular momentum is very low. These simple but realistic assumptions allow us to obtain the upper limit of the accretion rate of the flow to be

$$
\begin{aligned}
\dot{M} & \sim(3.5 \pm 0.58) \times 10^{14} x^{5 / 2} \mathrm{~g} / \mathrm{s} \\
& =(5.5 \pm 0.91) \times 10^{-12} x^{5 / 2} M_{\odot} \mathrm{yr}^{-1} .
\end{aligned}
$$

Here $x=\frac{r}{r_{\mathrm{g}}}$, is the dimensionless distance of the flaring region from the center. From transonic flow models (Chakrabarti 1990), the flow is expected to be supersonic only around $x \sim 2-3$ before disappearing into the black hole. This gives the accretion rate of A0620-00 in the quiescent state to be

$\dot{M}=(8.5 \pm 1.4) \times 10^{-11}\left(\frac{x}{3}\right)^{5 / 2} M_{\odot} \mathrm{yr}^{-1}$.

This is consistent with that reported by McClintock \& Remillard (2000) on the basis of X-ray observations. It is to be noted that Duldig et al. (1979) found a flux of $44 \pm 14 \mathrm{mJy}$ well after the outburst and concluded that intermittent emissions are possible and that mass transfer continues even in quiescence states. Our result also verifies such an assertion.

The procedure we have suggested here is general and should be applicable to determine the mass of black holes if the distance is reasonably well known. The only condition is that a hot, sub-Keplerian component should be present.

Acknowledgements. We thank the staff of the GMRT who have helped us to make this observation possible. GMRT is run by the National Centre for Radio Astrophysics of the Tata Institute of Fundamental Research. S.P. thanks a CSIR Fellowship which supported his work at the Centre for Space Physics. 


\section{References}

Chakrabarti, S. K. 1990, Theory of Transonic Astrophysical Flows (Singapore: World Scientific)

Davis, R. J., Edwards, M. R., Morison, I., \& Spencer, R. E. 1975, Nature, 257, 659

de Kool, M. 1988, ApJ, 334, 336

Duldig, M. L., Greenhill, J. G., Thomas, R. M., et al. 1979, MNRAS, 187,567

Elvis, M., Page, C. G., Pounds, K. A., Ricketts, M. J., \& Turner, M. J. L. 1975, Nature, 257, 656

Gelino, D. M., Harrison, T. E., \& Orosz, J. A. 2001, AJ, 122, 2668
Kuulkers, E., Fender, R. P., Spencer, R. E., Davis, R. J., \& Morison, I. 1999, MNRAS, 306, 919

Lasota, J.-P. 2001, New Astron. Rev., 45, 449

McClintock, J. E., Petro, L. D., Remillard, R. A., \& Ricker, G. R. 1983, ApJ, 266, L27

McClintock, J. E., \& Remillard, R. A. 1986, ApJ, 308, 110

McClintock, J. E., \& Remillard, R. A. 2000, ApJ, 531, 956

Owen, F. N., Balonek, T. J., Dickey, J., Terzian, Y., \& Gottesman, S. T. 1976, ApJ, 203, L15

Rees, M. 1984, ARA\&A, 22, 471

Shahbaz, T., Naylor, T., \& Charles, P. A. 1994, MNRAS, 268, 756 